Short reports

\title{
Solitary ileocolic arteriovenous malformation: Spongostan and silk therapeutic embolisation
}

\author{
M Alcalde, G Jiménez, P Díaz, M García-Díaz, JM Pascasio, R Moro, J Zapata, \\ A López-Santamaría
}

\section{Summary}

A debilitated patient with liver cirrhosis and poor haemostasis had a severe lower gastrointestinal haemorrhage. A superior mesenteric arteriogram revealed an early persistent and promiment draining vein in the ileocolic artery. Two fragments of Spongostan and silk were used to embolise the bleeding artery and the haemorrhage ceased immediately. No infarction of the embolised area was observed and the bleeding was controlled.

Keywords: arteriovenous malformation, ileocolic artery, embolisation, Spongostan, silk

Lower gastrointestinal haemorrhage, defined as bleeding distal to the ligament of Treitz, is most common in patients over 50 years old. Several causes are implicated, of which diverticulosis is by far the most frequent (box 1). ${ }^{1}$ Most episodes stop spontaneously, although minor bleeding frequently re-occurs which is controlled by nonsurgical means. The bleeding source is commonly in the right side of the colon, an area that may be difficult to reach endoscopically. ${ }^{2}$ Angiography is very useful in localisation, diagnosis and treatment. ${ }^{3}$ We report a patient with lower gastrointestinal haemorrhage due to arteriovenous malformation, who was diagnosed and treated with angiographic embolisation.

\section{Case report}

Infanta Cristina

University Hospital of Badajoz,

Carretera de

Portugal s/n 06080

Badajoz, Spain

Department of

Gastroenterology

$M$ Alcalde

G Jiménez

P Díaz

M García-Díaz

JM Pascasio

Department of

Radiology

R Moro

J Zapata

A López-Santamaría

Correspondence to Dra. Mar Alcalde Rubio, C/ Francisco Pizarro, no 19, $2^{\circ}$, Badajoz 06001, Spain

A 62-year-old woman was admitted because of gastrointestinal haemorrhage. Her clinical history revealed long-standing liver cirrhosis with positive $\mathrm{HBs} \mathrm{Ag}$ and Child-Pugh score parameters of B. The patient had an episode of upper gastrointestinal haemorrhage because of oesophageal varices a year before the current admission. Significant admission laboratory tests revealed: haemoglobin $156 \mathrm{~g} / \mathrm{l}$, haematocrit 0.30 , total white cell $1.4 \times 10^{9} / 1$, platelets $41 \times 10^{9} /$, blood urea nitrogen $34 \mathrm{mg} / \mathrm{dl}$, total bilirubin $28 \mathrm{mmol} / \mathrm{l}$, lactate dehydrogenase $463 \mathrm{IU} / 1$, aspartate transaminase $37 \mathrm{IU} / 1$, alanine transaminase $28 \mathrm{IU} / \mathrm{l}$, prothrombin activity $47 \%$. Upper gastrointestinal endoscopy showed gastro-oesphageal varices with no stigmata of bleeding, and no blood was found in the stomach and duodenum. Colonoscopy showed a large quantity of blood in the colon. The bleeding site could not be determined.
Fresh red blood was found in the caecum and terminal ileus. Superior mesenteric arteriogram (figure 1) demonstrated an early persistent and prominent draining vein in the ileocolic artery. On the seventh hospital day and after several episodes of lower gastrointestinal haemorrhage in small quantities, the patient presented severe rectal bleeding. A subselective catheterisation of a second order intestinal branch of the ileocolic artery was performed. Selective intraarterial embolisation was done as an emergency therapy with Spongostan ${ }^{\circledR}$ (Ferrosan, Denmark) and silk (Mersilk ${ }^{\circledR}$ 5/0, Johnson \& Johnson, Ethicon, UK) using two pieces $(2 \times 2 \mathrm{~mm})$ of each. Postembolisation arteriography showed that the early persistent prominent draining vein was no longer opacified (figure 2). Over the next four days the patient was closely observed for signs of peritoneal irritation, which would suggest bowel infarction but no signs were detected. No recurrence of

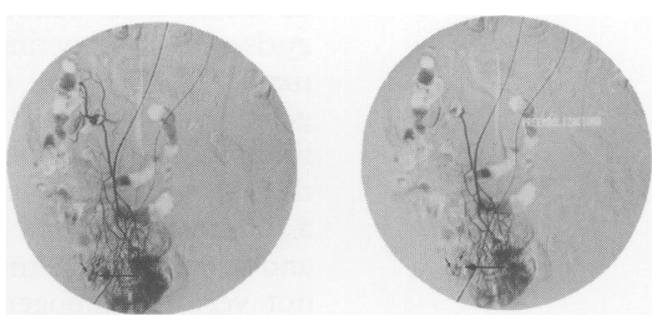

Figure 1 Pre-embolisation arteriogram (A) Arterial phase of a superior mesenteric artery injection shows early opacification of a vein adjacent to a distal second branch of the ileocolic artery (arrow); (B) venous phase shows a persistent and promiment draining vein filled early in the arterial phase
Figure 2 Postembolisation arteriogram (A) Arterial phase of a superior mesenteric artery injection shows occlusion of one of the distal second branches of the ileocolic artery and the persistent prominent draining vein is no longer opacified; (B) venous phase shows that the persistent prominent vein is no longer opacified 
the bleeding was observed after therapy and the patient remained asymptomatic six months later.

\section{Discussion}

Upper gastrointestinal haemorrhage is generally acute and of a magnitude and severity that requires hospitalisation. On the other hand, lower gastrointestinal haemorrhage is more often of a chronic, intermittent nature. For the most part of it is not immediately lifethreatening. The bleeding in $20-30 \%$ of patients continues and recurs. Colonoscopy is often prevented by large quantities of intraluminal blood. Also, the bleeding sources are commonly in the right side of the colon, which is difficult to reach endoscopically. ${ }^{2}$ Selective angiography of the superior mesenteric artery is generally performed when colonoscopy fails. The conventional film screen angiography can detect bleeding at a rate as low as $1-1.5 \mathrm{ml} /$ min. ${ }^{1}$ The sine qua non for active haemorhage is detection of extravasation of contrast material into the lumen of the gut, which is difficult to see on angiography because of intermittent bleeding. ${ }^{1}$ In elderly or debilitated patients who are the most frequent sufferers from lower gastrointestinal haemorrhage and, as in our case, those with a poor haemostasis, embolisation can be the chosen therapeutic procedure. Other procedures used are selective infusion of vasopressin and, recently, laser photocoagulation. $^{4}$

Numerous agents have been employed as emboli such as Ivalon, Gelfoam, Spongostan, silk, Gianturco coils, isobutyl-2-cyanoacrylate or detachable balloons. They can be categorised as short-acting, intermediate or permanent. ${ }^{2}$ Spongostan or Gelfoam (absorbable gelatin sponge) are the most widely used intermediate agents. Spongostan is highly thrombogenic and was originally developed as a haemostatic agent. Silk is not biodegradable and so produces permanent obstruction. Silk is not very thrombogenic and obstruction may not form promptly after embolisation. For that reason, Spongostan and silk are used together; silk holding the Spongostan in place temporarily, preventing distal embolisation of fragments and assuring permanent obstruction once thrombosis develops. Of the other embolic materials used, Gianturco coils or Ivalon provide permanent occlusion, isobutyl-2-cyanoacrylate may glue the catheter and is therefore difficult to handle, and detachable balloons are expensive and difficult to position.

1 Zuckerman D, Bocchini T, Birnbaum E. Massive hemorrhage in the lower gastrointestinal tract in adults: diagnostic imaging and intervention $A f R$ 1993; 161: 703-11.

2 Sebrechts C, Bookstein J. Embolization in the management of lower-gastrointestinal hemorrhage. Semin Intervent Radiol 1988; 5: $39-48$.

3 Doemeny J, Baum S. Angiographic diagnosis in acute gastrointestinal hemorrhage. Semin Intervent Radiol 1988; 5: 1-9.
Lower gastrointestinal haemorrhage: causes

- diverticulosis

- angiodysplasia

- arteriovenous malformation

- polypectomy

- telangiectasia

- inflammatory bowel disease

- colitis (ischaemic or infectious)

- colonic neoplasm

- ulcers (rectal or uraemic stress)

- colorectal haemangioma

Box 1

\section{Summary point}

Embolotherapy is a useful option in the management of severe lower gastrointestinal

haemorrhage in patients at high risk for surgery

Box 2

The number of Spongostan fragments that should be used is unclear. We used two Spongostan and silk particles, about $2 \times$ $2 \mathrm{~mm}$, which were placed in dilute contrast medium and deposited as distally as possible into the second branch of the ileocolic artery. Repeated flushing of the embolic material and contrast were performed until the persistent draining vein was no longer opacified. The most feared complication after embolisation is irreversible ischaemia with resultant bowel infarction or chronic stricture. Such a risk can be diminished by using the least amount of embolic material and by depositing the agents as distally as possible in the artery branch affected. This risk is higher in small bowel than in large bowel because of the greater and more tenous collateral potential. ${ }^{2}$ However, in some cases, small areas of ischaemia or infarction occur after embolisation; these are usually clinically well tolerated. Patients should be closely observed for several days after embolisation in search of signs of peritoneal irritation suggesting bowel ischaemia. ${ }^{5,6}$ Embolotherapy provides control of the bleeding in 80 to $100 \%$ of cases and rebleeding is rare. ${ }^{2}$ In the patient reported, haemostasis was achieved rapidly following embolisation without ischaemic changes to the embolised bowel. No recurrence of haemorrhage occurred in six months of follow-up.

4 Helmrich GA, Stallworth JR, Brown JJ. Angiodysplasia: characterization, diagnosis, and advances in treatment. characterization, diagnosis, and

5 Palmaz J, Walter J, Kyung C. Therapeutic embolization of the small bowel arteries. Radiology 1984; 152: 377-82. Kandarpa K, Fellows K, Eralis A, Flores A. Solitary ileal malformation: preoperative localization by coli embolization. $A f R$ 1986; 146: 787-8. 